\title{
Water relations of the tos 1 tomato mutant at contrasting evaporative demand
}

\author{
Oliva Jurado ${ }^{\text {, Alfonso Albacete }}{ }^{\mathrm{b}}, \mathrm{M}$. Carmen Martínez-Ballesta ${ }^{\mathrm{b}}$, Micaela Carvajal ${ }^{\mathrm{b}}$, Francisco \\ Pérez-Alfoceab ${ }^{b}$ Ian C. Dodd ${ }^{c}$ and M. Remedios Romero-Aranda ${ }^{b, *}$ \\ aDepartamento de Mejora Vegetal, Estación Experimental La Mayora, Consejo Superior de Investigaciones Científicas, 29750 Algarrobo-Costa, \\ Málaga, Spain \\ ${ }^{\text {b}}$ Departamento de Nutrición Vegetal, Centro de Edafología y Biología Aplicada del Segura, Consejo Superior de Investigaciones Científicas, Campus \\ Universitario de Espinardo, 30100 Murcia, Spain \\ 'The Lancaster Environment Centre, Lancaster University, LA1 4YQ, UK
}

\author{
Correspondence \\ ${ }^{*}$ Corresponding author, \\ e-mail: rromero@eelm.csic.es \\ Received 3 March 2009; revised 28 May \\ 2009 \\ doi: 10.1111/j.1399-3054.2009.01259.x
}

The tos1 (tomato osmotically sensitive) mutant, isolated from an in vitro screen of root growth during osmotic stress, was less sensitive to exogenous ABA, but accumulated more ABA under osmotic stress than WT plants. We assessed growth and water relations characteristics of hydroponically grown tos 1 seedlings (in the absence of osmotic stress) at low and high evaporative demands. Growth of tos 1 was severely inhibited at both high and low evaporative demands. Twenty DAS, WT and tos 1 genotypes had a similar leaf water and turgor potential, but mature tos 1 plants (45 day old) showed a significant diurnal loss of leaf turgor, with recovery overnight. Increased evaporative demand increased turgor loss of tos 1 plants. High evaporative demand at the beginning of the day decreased stomatal conductance of tos1, without diurnal recovery, thus whole plant transpiration was decreased. De-topped tos 1 seedlings showed decreased root hydraulic conductance and had a 1.4-fold increase in root ABA concentration. Impaired root function of tos 1 plants failed to meet transpirational water demand and resulted in shoot turgor loss, stomatal closure and growth inhibition.

\section{Introduction}

Trans-gene and mutational analyses have both contributed to our understanding of the underlying mechanisms leading to drought stress adaptation (Chinnusamy et al. 2004). Nevertheless, for these genetic techniques to be fully exploited, it is necessary to assess the physiological mechanisms that are functional at the whole plant level in order to improve plant water status and consequently maximise growth under drought stress.

To avoid leaf dehydration under drought conditions, plants can develop three strategies: first, close stomata and decrease transpiration rate; second, decrease shoot growth in order to limit transpiration; and third, control root water uptake and/or plant water status via root growth and root hydraulic conductivity (Parent et al. 2009). It is widely accepted that ABA plays a role in drought stress responses, including gene expression, metabolic changes, stomatal closure and morphological changes (Bray 2002, Nejad and Meeteren 2007, Urano et al. 2009, Wilkinson and Davies 2002). However, ABA levels in plants fluctuate widely in response to environmental changes, especially drought stress (Seki et al. 2007). It has been hypothesised that at least four independent signal pathways function

Abbreviations - ABA, abcisic acid; DAS, days after sowing; PAR, photosynthetically active radiation; VPD, vapour pressure deficit; WT, wild-type. 
in the activation of stress-inducible genes under dehydration conditions: two ABA-dependent and two ABA-independent pathways (Shinozaki and YamaguchiShinozaki 2007). On the other hand, root hydraulic conductance and transpiration rate are key parameters contributing to plant water uptake and plant water status (Steudle and Peterson 1998) and both processes can be modified by atmospheric evaporative water demand (Javot and Maurel 2002, Mott et al. 1997). Plant morphological traits, such as stomatal density, foliar epicuticular wax load and size of the root system, may respond to changes in shoot water status and have been related to endogenous $\mathrm{ABA}$ and plant adaptation to drought (Lake and Woodward 2008).

The tomato mutant, tos1 (tomato osmotically sensitive), was obtained by screening seedling root growth to osmotic stress in a population of mutagenised tomato seeds (Borsani et al. 2002). In vitro assays showed that tos1 seedlings (7 DAS) accumulated more ABA than the WT after osmotic stress, yet root (and callus) growth was less sensitive to exogenous ABA in the absence of osmotic stress (Borsani et al. 2002). Preliminary growth analyses under Mediterranean greenhouse conditions showed that tos 1 grew less than WT plants in all developmental stages, and mature tos 1 plants wilted during the day but recovered turgor overnight. Thus, the tos 1 phenotype could be related to a high transpirational water loss (perhaps related to ABA insensitivity) and/or to a low capacity of plants to take up water from the roots. Other plant adaptations such as osmotic adjustment, changes in stomatal density or epicuticular wax synthesis may also be altered in this mutant, although it can be difficult to establish whether these are directly caused by the tos 1 mutation or secondarily induced by water stress. Therefore, the aim of this study was to understand the mechanisms that mediate the growth inhibition and wilty phenotype of tos1.

\section{Materials and methods}

\section{Plant material and growth conditions}

WT (Solanum lycopersicum cv. Moneymaker) and tos 1 mutant tomato plants were grown hydroponically under high (Experiment 1) and low (Experiment 2) evaporative water demand.

Experiment 1 (high VPD) was developed in a plastic greenhouse, under the natural conditions of the Southeast Spanish Mediterranean area during late spring and the beginning of the summer. Humidity, air temperature and radiation were recorded continuously by a data logger (Meteodata-256, Geonica, Madrid, Spain). During the experimental period, minimum/maximum average air temperatures were $19 / 35^{\circ} \mathrm{C}$ and the relative humidity ranged between 20 and $95 \%$. The average maximum PAR $(400-700 \mathrm{~nm})$ at midday was $920 \mu \mathrm{mol} \mathrm{m}^{-2} \mathrm{~s}^{-1}$. For a period of 2 months, 80 plants (40 per genotype) were grown in 10-I plastic pots filled with continuously aerated Hoagland nutrient solution, which was changed weekly. There was one plant per pot. Experiment 2 (low VPD) was developed in a growth chamber (Model Fitoclima $27000 \mathrm{EHHF}, \mathrm{ARALAB}$, Lisbon, Portugal) that allowed daily cycles of air temperature, relative humidity and radiation similar to outdoors. The environmental conditions in this experiment were those typical of the beginning of spring, which were estimated from average climatic data recorded inside the greenhouse over the last 10 years. Humidity, air temperature and radiation were recorded continuously by the growth chamber's control system. Minimum/maximum air temperatures were $16 / 28^{\circ} \mathrm{C}$ and the relative humidity ranged between 40 and $95 \%$. Maximum PAR at midday was set at $750 \mu \mathrm{mol} \mathrm{m} \mathrm{m}^{-2} \mathrm{~s}^{-1}$. Thirty plants (15 per genotype) were grown as in Experiment 1 and an additional set of twenty plants (10 per genotype) were grown individually in 3-I plastic pots in order to determine plant water uptake by weight loss.

Ranges of relative humidity have been defined in terms of atmospheric VPD, because it determines the water demand in the atmosphere and the water vapour difference between roots and leaves (Grange and Hand 1987). In this study, relative humidity data were used to calculate $\mathrm{VPD}$ as $\mathrm{VPD}=e_{\mathrm{sat}}\left(T_{\text {air }}\right)-e_{\mathrm{a}}$; $e_{\mathrm{a}}=\left(\mathrm{RH} \times e_{\text {sat }}\right) / 100$, where $e_{\text {sat }}$ is the saturating vapour pressure at a particular air temperature, $e_{\mathrm{a}}$ the actual air vapour pressure and $\mathrm{RH}$ the relative humidity. Fig. 1 shows representative daily evolution of VPD during Experiments 1 and 2 with average midday values of 3.4 and $1.8 \mathrm{kPa}$, respectively.

\section{Plant water status}

Fully expanded leaves at the five to seven leaf positions from the top of the plant were used to determine plant water relations parameters. Leaf water potential $(\Psi \mathrm{w})$ was determined in the morning and at midday on 20- and 45-day-old plants, with a Scholander chamber (Soil Moisture Equipment Corp., Santa Barbara, CA). Six leaves per genotype were sampled each time. After $\Psi \mathrm{w}$ was determined, leaf mid-veins were removed from adjacent leaves and the leaves were placed in $5 \mathrm{ml}$ pipette tips sealed in eppendorf tubes and stored at $-20^{\circ} \mathrm{C}$ for further analysis. The frozen samples were allowed to thaw at room temperature then centrifuged at $4000 \mathrm{~g}$ for $5 \mathrm{~min}$ to extract the cell sap. The osmolarity of the collected sap $(\Psi \pi)$ was determined with a dew point 


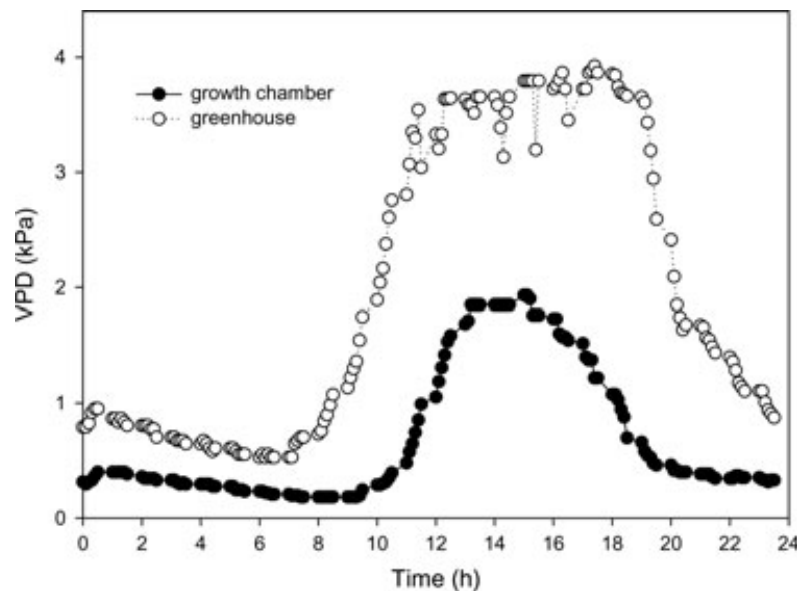

Fig. 1. Typical pattern of atmospheric VPD inside the greenhouse (Experiment 1) and inside the growth chamber (Experiment 2).

osmometer (Model 5520, Wescor, Logan, UT). Turgor potential $(\Psi p)$ was estimated as the difference between $\Psi \mathrm{W}$ and $\Psi \pi$.

\section{Gas exchange measurements}

Stomatal conductance (gs) and transpiration rate (E) were measured in young (20 DAS) and mature (45 DAS) plants with a portable $\mathrm{CO}_{2} / \mathrm{H}_{2} \mathrm{O}$ analyser (Model LI-6400, LiCor Inc., Lincoln, NE). A LED light source was attached in the leaf chamber, artificially controlling the PAR to the same values in the greenhouse or growth chamber. Six plants (two leaves per plant) were measured in each genotype.

\section{Plant water uptake, osmotic root hydraulic conductance and $\mathrm{ABA}$ analysis}

Plant water uptake was determined in young plants grown under low VPD in the growth chamber, as the difference in pot weight every 2 days. Osmotic root hydraulic conductance was measured in detached root systems that exuded water under atmospheric pressure (Aroca et al. 2006). Two hours after the lights were turned on, the same plants used to determine water uptake were cut below the cotyledons and a silicone tube was attached to the stem. Sap exuded during the first $10 \mathrm{~min}$ was discarded and the exudation during the following $1 \mathrm{~h}$ was collected with a syringe and weighed. The osmolarities of the exuded sap and the nutrient solution were determined using an osmometer (Model 5520, Wescor, Logan, UT). Osmotic root hydraulic conductance $(\mathrm{L})$ was calculated as $\mathrm{L}=\mathrm{Jv} / \Delta \Psi$, where $\mathrm{J} v$ is the exuded sap flow rate $(\mathrm{J} v=\mathrm{g}$ xylem sap exudate $\mathrm{g}^{-1}$ root $\mathrm{DW} \mathrm{h}^{-1}$ ) and $\Delta \Psi$ is the osmotic potential difference between the exuded sap and nutrient solution. ABA concentrations of root tissue and root sap exudates were determined as in Ghanem et al. (2008).

\section{Leaf morphological traits}

Morphological traits were studied only on mature 60day-old plants. Leaf impressions were obtained from both surfaces of the basal leaflet using the silicon leaf impression method (Romero-Aranda et al. 2001). Impressions were examined with a light microscope at $400 \times$ magnification, and stomata were counted on 10 fields taken at random from each sample. Five leaves per genotype were sampled in each experiment.

Foliar epicuticular wax content was assessed (Ebercon et al. 1977). In each experiment, five samples per genotype were collected; each individual sample consisted of 10-12 leaflets from the same plant. Leaf area was determined and epicuticular wax was then extracted by submerging the leaflets, for 20-25 s, into $25 \mathrm{ml}$ of chloroform, with agitation, at room temperature. The extracts were filtered and evaporated to dryness. Total wax content was determined by weighing the residue with an analytical balance and quantities expressed as mass of wax per unit leaf area.

\section{Growth measurements}

Growth parameters were determined in young (30 DAS) and mature (60 DAS) plants grown under low and high VPD. Plant height and stem diameter were recorded on six randomly selected plants per genotype. Plants were harvested and divided into leaves, stems and roots and their fresh and dry weights recorded. Leaf area was measured using a Leaf Area Meter $(\Delta \mathrm{T}$ Devices Ltd., Cambridge, UK).

\section{Statistics}

Data were subjected to analysis of variance (ANOVA) using SPSS/PC+ Statistics software package (SPSS 15.0, standard version 2006 SPSS, Inc. Chicago, IL). Means were separated with the Student-Newman-Keuls $(\mathrm{S}-\mathrm{N}-\mathrm{K})$ test at $P<0.05$.

\section{Results}

No leaf wilting was observed in young tos1 plants; however, mature tos1 plants wilted during the day and wilty symptoms varied with evaporative demand. Mature tos1 plants grown under low VPD showed leaf wilting at the beginning of the afternoon. This wilting was observed only in young leaves at the top of the plant and leaves 

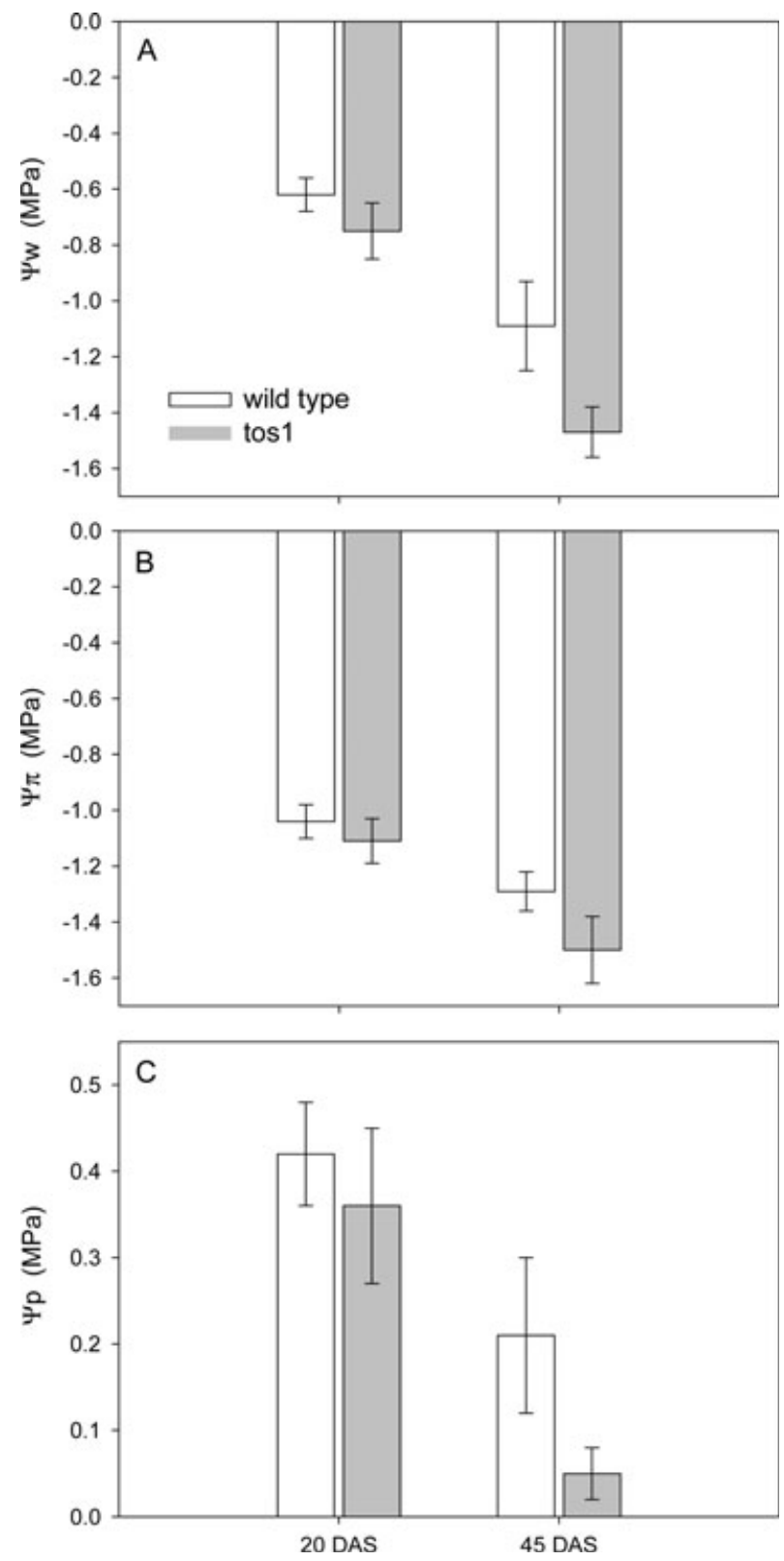

Fig. 2. Leaf water $(\Psi W)$, leaf osmotic $(\Psi \pi)$, and leaf turgor $(\Psi p)$ potentials recorded at midday in young (20 DAS) and mature (45 DAS) plants grown under high VPD. Values are means $(n=6)$ and vertical bars indicate $\pm \mathrm{SE}$.

recovered turgor overnight. Mature tos 1 plants grown under high VPD showed leaf wilting in most of the plant from early in the morning until the end of the photoperiod but again recovered turgor overnight.

Young plants (20 DAS) showed no genotypic differences in leaf water potential $(\Psi \mathrm{w})$, leaf osmotic potential $(\Psi \pi)$ and leaf turgor potential ( $\Psi$ p) (Fig. 2A-C). However, mature tos 1 plants (45 DAS) showed significantly lower leaf $\Psi \mathrm{w}$, leaf $\Psi \pi$ and leaf $\Psi p$ than
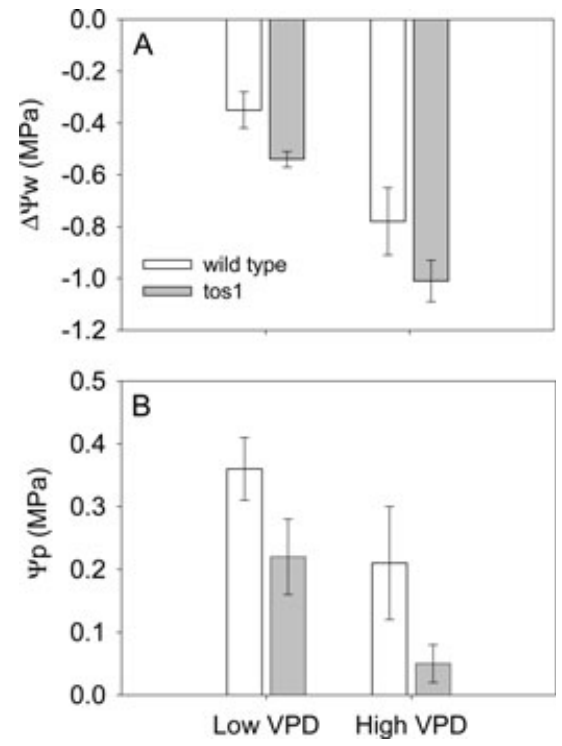

Fig. 3. Decrease of leaf water potential from morning to midday $(\Delta \Psi w)$ and midday leaf turgor potential $(\Psi p)$ of 45 -day-old plants grown under low or high VPD. Values are means $(n=6)$ and vertical bars indicate $\pm \mathrm{SE}$.

mature WT plants. Mature tos 1 plants showed a greater decrease in leaf $\Psi \mathrm{w}$ from morning to midday than mature WT plants at both VPDs (Fig. 3A). Although midday leaf $\Psi p$ of both genotypes was higher at low VPD, $\Psi$ p was lower in mature tos1 than in WT plants regardless of VPD (Fig. 3B).

Young WT and tos 1 plants had similar midday rates of stomatal conductance and transpiration averaging $0.45 \mathrm{~mol} \mathrm{~m}^{-2} \mathrm{~s}^{-1}$ and $6.4 \mathrm{mmol} \mathrm{m} \mathrm{m} \mathrm{s}^{-1}$, respectively. However, mature tos 1 had significantly lower gs and transpiration than WT under both low and high VPD (Fig. 4). Transpiration rates of WT plants were higher at high VPD than at low VPD but transpiration was not affected by VPD in tos 1 plants. At high VPD, gs and $\mathrm{E}$ of both genotypes increased to maximal values by 10:00 $\mathrm{h}$ in response to increasing irradiance (Fig. 5A, B). Thereafter, WT plants maintained higher gs and $\mathrm{E}$ values during the day while the same parameters decreased substantially in tos 1 such that stomata remained almost closed all day long.

Plant water content (FW-DW) of tos1 plants grown under low and high VPD was 42 and 35\%, respectively, lower than in WT plants. Daily plant water uptake in tos 1 plants was lower than in WT plants, and this genotypic difference increased with plant development (Fig. 6) as a result of decreased growth of tos 1 plants. At low VPD, all growth parameters were significantly lower in tos 1 than in WT plants and high VPD further decreased tos 1 growth (Table 1). Compared with plants grown at low VPD, high VPD decreased total biomass of WT and 

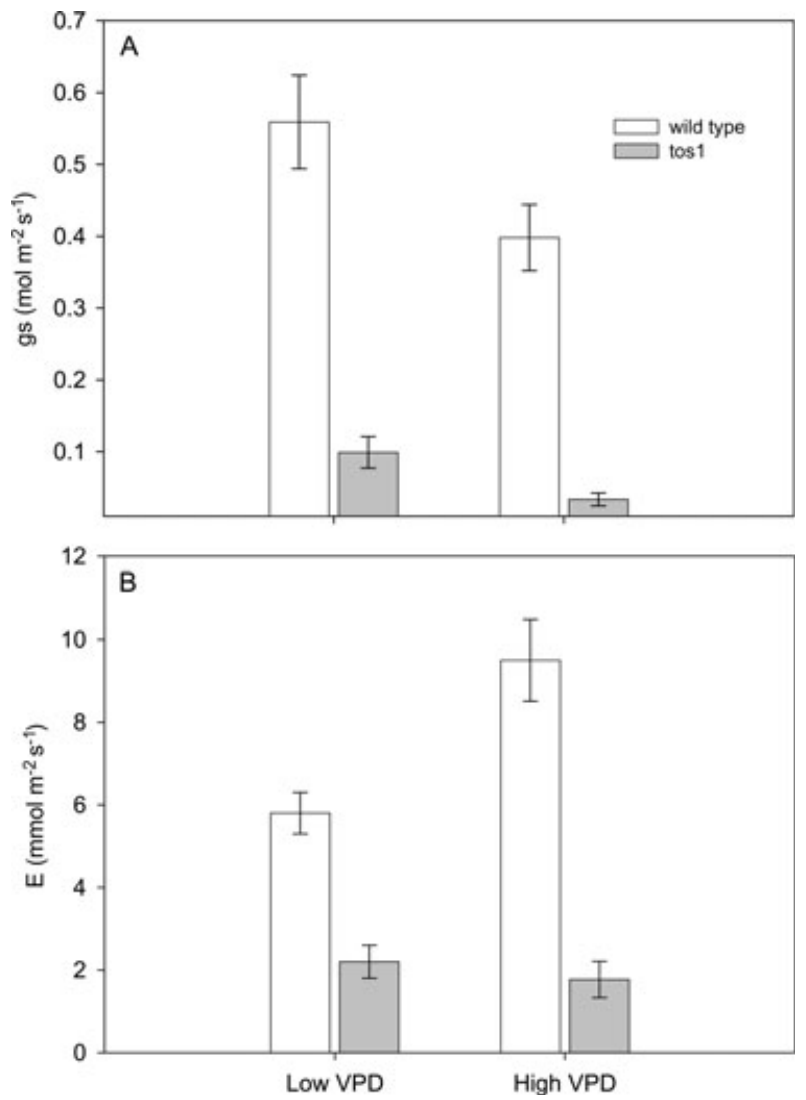

Fig. 4. Midday stomatal conductance (gs) and transpiration rates (E) of 45-day-old plants grown under low or high VPD. Values are means $(n=12)$ and vertical bars indicate \pm SE.

tos 1 plants by 14 and $39 \%$, respectively. High VPD also reduced leaf area by 40 and $80 \%$ in WT and tos 1 , respectively, and plant height by 42 and $51 \%$ in WT and tos 1 , respectively.

Distribution of biomass between the different plant organs varied with the genotype and plant age (Fig. 7). Root to shoot ratio in young plants (20 DAS) was 0.23 and 0.59 for WT and tos 1 plants, respectively. On the other hand, percentages of dry weight of roots, stems and leaves were 20, 29, and 51\% in WT plants and 29, 29 , and $43 \%$ in tos 1 plants, all respective to the whole plant's dry weight (Fig. 7A). A significant reduction in all plants's organs was observed in mature tos 1 plants (Fig. 7B), but the root to shoot ratio of these mature tos 1 plants was still higher $(0.32 \pm 0.04)$ than WT plants $(0.12 \pm 0.00)$, confirming the higher root to shoot ratio found in young tos 1 plants.

In plants grown under low VPD, root exudation rate and the osmotic gradient between nutrient solution and sap exudates were lower in tos 1 plants (Fig. 8A, B) causing lower osmotic root hydraulic conductance (Fig. 8C). Concentrations of $\mathrm{ABA}$ in the roots $\left(28.8 \pm 6.1 \mu \mathrm{g} \mathrm{g} \mathrm{g}^{-1}\right.$
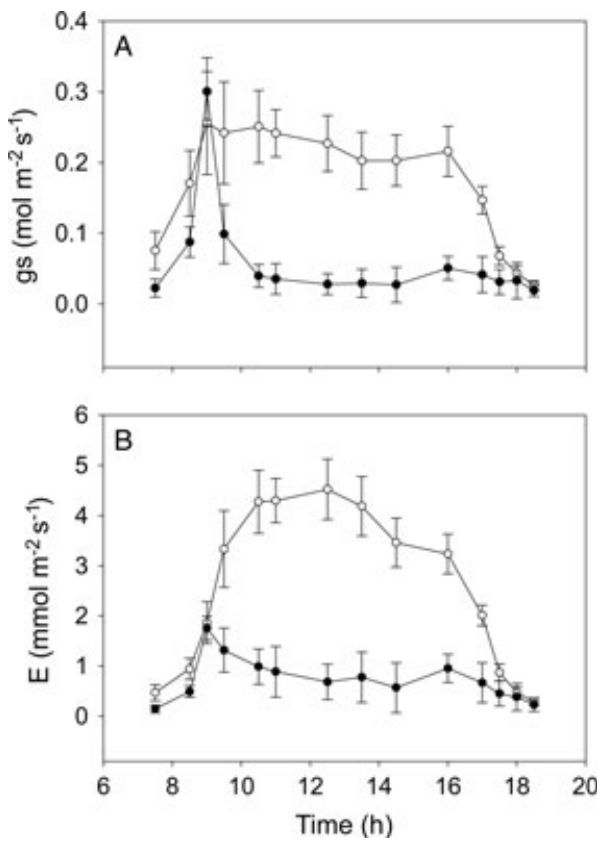

Fig. 5. Daytime stomatal conductance (gs) and transpiration (E) of 45day-old plants grown under high VPD. WT genotype (open circles) and tos 1 genotype (closed circles). Values are means $(n=12)$ and vertical bars indicate \pm SE.

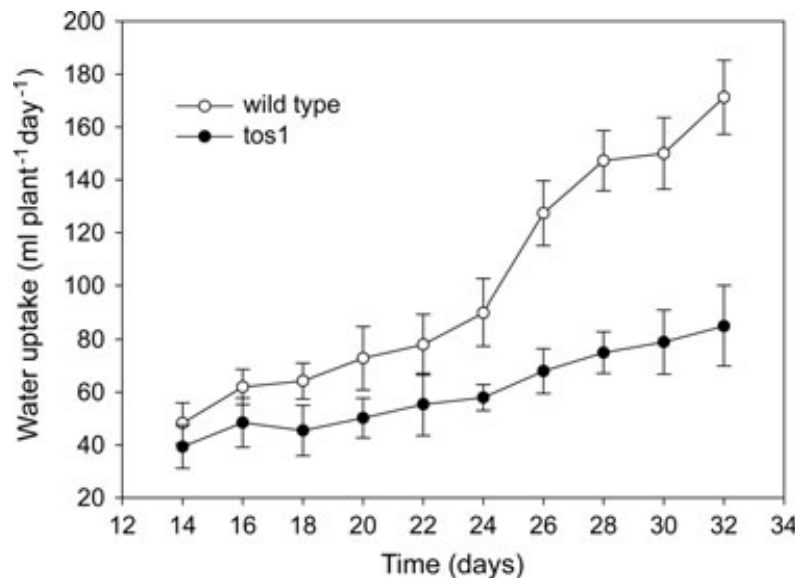

Fig. 6. Daily plant water uptake of young WT and tos 1 plants grown under low VPD. Values are means $(n=6)$ vertical bars indicate \pm SE.

FW) and xylem sap exudate $\left(0.186 \pm 0.018 \mu \mathrm{mol} \mathrm{m}{ }^{-3}\right)$ of tos 1 were significantly higher than that of WT plants $\left(17.3 \pm 1.6 \mu \mathrm{g} \mathrm{g}^{-1} \mathrm{FW}\right.$ and $0.136 \pm 0.018 \mu \mathrm{mol} \mathrm{m} \mathrm{m}^{-3}$ in root tissue and xylem sap exudates, respectively).

Although there were no genotypic differences in stomatal density and foliar epicuticular wax load under low VPD, under high VPD stomatal density was significantly lower in tos 1 than in WT plants, whereas 
Table 1. Growth parameters of mature WT and tos 1 plants grown under low and high VPD. Values are mean \pm SD of six plants. Letters indicate statistically significant difference according to Student-Newman-Keuls $(\mathrm{S}-\mathrm{N}-\mathrm{K})$ test at $P<0.05$.

\begin{tabular}{|c|c|c|c|c|}
\hline & & $\begin{array}{c}\text { Fresh weight } \\
(\mathrm{g})\end{array}$ & $\begin{array}{l}\text { Leaf area } \\
\qquad\left(\mathrm{cm}^{2}\right)\end{array}$ & $\begin{array}{c}\text { Plant height } \\
(\mathrm{cm})\end{array}$ \\
\hline \multirow[t]{2}{*}{ Low VPD } & WT & $486 \pm 50 a$ & $4790 \pm 781 a$ & $90 \pm 5 a$ \\
\hline & tos1 & $143 \pm 27 c$ & $2010 \pm 384 c$ & $61 \pm 10 b$ \\
\hline \multirow[t]{2}{*}{ High VPD } & WT & $417 \pm 25 b$ & $2723 \pm 137 b$ & $52 \pm 3 b$ \\
\hline & tos1 & $88 \pm 17 d$ & $371 \pm 93 d$ & $30 \pm 3 c$ \\
\hline
\end{tabular}
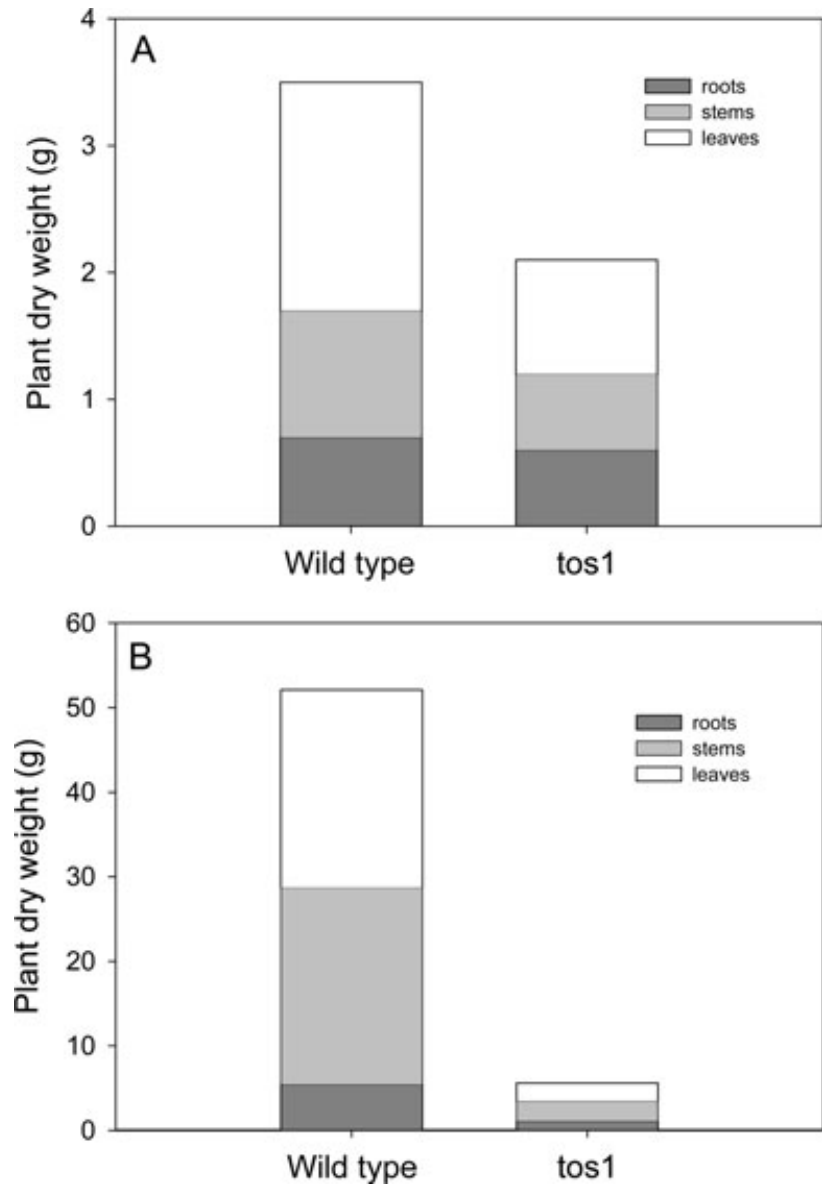

Fig. 7. Dry weight distribution between leaves stems and roots of 20day-old (A) and 45-day-old (B) plants grown in the greenhouse during Experiment 1. Values are means $(n=6)$.

foliar epicuticular wax load was significantly higher in tos1 than in WT plants (Table 2).

\section{Discussion}

The tos1 mutant was less able to maintain leaf turgor in mature (45-day-old) plants grown at low VPD (Fig. 3)
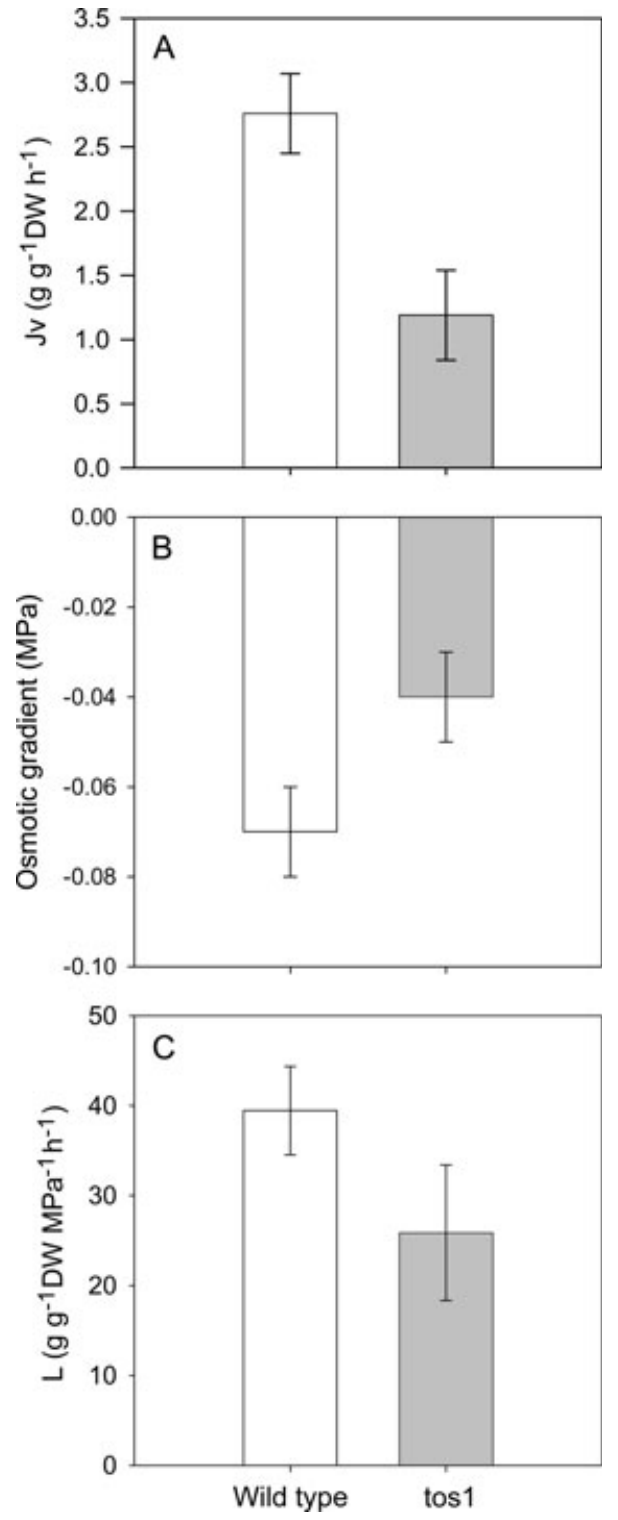

Fig. 8. Root sap flow (Jv), osmotic gradient and root osmotic conductance (L) of 20-day-old plants grown under low VPD. Values are means $(n=6)$ and the vertical bars indicate \pm SE.

or plants grown under high VPD in typical Mediterranean greenhouse conditions (Fig. 2). Leaves of tos 1 plants began to lose turgor at leaf $\Psi_{\mathrm{w}}$ ranging from -1.0 to $-1.2 \mathrm{MPa}$. These values are not unusual in Mediterranean greenhouse culture of commercial tomato cultivars free from leaf wilting symptoms (Romero-Aranda et al. 2001, 2002). It was important to resolve whether this was because of an inability of tos1 leaves to limit water loss, or an inability of the root system to supply enough water to meet transpirational demand.

Leaves from tos1 plants grown at high VPD showed anatomical alterations (lower stomatal density and 
Table 2. Stomatal density and foliar epicuticular wax content recorded in mature WT and tos1 plants grown under low and high VPD. Values are mean $\pm S D$ of five plants. Letters indicate statistically significant difference according to Student-Newman-Keuls (S-N-K) test at $P<0.05$.

\begin{tabular}{llrr}
\hline & & $\begin{array}{l}\text { Stomatal density } \\
\left(\text { number } \mathrm{mm}^{-2}\right)\end{array}$ & \multicolumn{1}{c}{$\begin{array}{c}\text { Foliar wax } \\
\left(\mu \mathrm{gm}^{-2}\right)\end{array}$} \\
\hline Low VPD & WT & $187 \pm 21 \mathrm{~b}$ & $8.94 \pm 1.13 \mathrm{~b}$ \\
& tos1 & $171 \pm 17 \mathrm{~b}$ & $10.74 \pm 1.82 \mathrm{~b}$ \\
High VPD & WT & $229 \pm 25 \mathrm{a}$ & $9.70 \pm 1.69 \mathrm{~b}$ \\
& tos1 & $133 \pm 10 \mathrm{c}$ & $16.40 \pm 1.97 \mathrm{a}$ \\
\hline
\end{tabular}

higher foliar epicuticular wax load than WT plants -Table 2) that would have acted to restrict water loss. Although elevated tissue ABA concentration alone has been shown to promote developmental changes in leaf anatomy (decreased stomatal density) that mimic the effects of water deficit (Lake and Woodward, 2008), these responses were detected only at high VPD (Table 2), whereas tos1 ABA status was increased even at low VPD, which suggests an adaptation to ambient drought rather than a direct effect of elevated $A B A$ status.

Although stomatal conductance and transpiration rate of tos1 was similar to WT plants early in the day (Fig. 5), stomatal conductance of tos 1 plants grown in the greenhouse abruptly declined after 09:30 h when atmospheric VPD had reached 2 kPa (Fig. 1). Thus tos1 stomata remained almost closed throughout the day in plants grown at high VPD (Fig. 5A), and even plants grown at low VPD showed severe midday stomatal closure (Fig. 4), again when atmospheric VPD had reached $2 \mathrm{kPa}$ (Fig. 1). Although this stomatal closure decreased plant transpiration (Fig. 4B, 5B), it did not prevent turgor loss of tos1 plants (Fig. 2C, 3B).

Several possibilities could account for the sensitive stomatal closure of tos1. Although our stomatal and leaf water relations were made with insufficient temporal resolution to distinguish cause and effect, it is possible that decreased leaf turgor directly closed tos 1 stomata, an hypothesis that is best tested by measuring stomatal conductance of plants grown with root pressurisation to counteract the decrease in turgor (Gollan et al. 1986). Similar experiments with plants grown in drying soil have demonstrated that root pressurisation can prevent (Yao et al. 2001) or have no effect (Gollan et al. 1986) on drought-induced stomatal closure; the latter response emphasising the role of chemical signals (such as ABA) in stomatal closure (Dodd 2005). Although xylem sap collected from de-topped roots of tos 1 had a 37\% higher ABA concentration, this may have been a result of the much slower $(65 \%)$ exudate flow rate from tos 1 plants (Fig. 8A). However, root ABA concentrations of tos1 were also $66 \%$ higher than in WT plants even in plants grown under low VPD, possibly reflecting increased leaf ABA synthesis in response to turgor loss (Pierce and Raschke 1980). Assuming that tos1 stomatal closure was ABA mediated, it is possible that decreased leaf water potential and turgor (Fig. 2C, $3 \mathrm{~B}$ ) increased stomatal sensitivity to ABA (Hose et al. 2000, Karbulková et al. 2008, Tardieu and Davies 1992) causing stomatal closure, a suggestion that is at odds with in vitro root growth data showing that tos 1 is $A B A$ insensitive (Borsani et al. 2002). Paired measurements of stomatal conductance and xylem sap ABA concentration in vivo (Vysotskaya et al. 2008) are necessary to resolve whether tos 1 shows stomatal insensitivity to ABA.

Regardless of the mechanisms causing stomatal closure of tos 1 , this would act to sustain water status, thus focusing attention on the ability of the root system to supply sufficient water to match transpirational demand. Clearly the higher root to shoot ratio of tos 1 plants (Fig. 7) was insufficient to prevent turgor loss, although the reduction of root to shoot ratio with plant development (cf. Fig. 7A, B) could have exacerbated the negative water balance of more mature tos1 plants (Fig. 2).

Both transpirational and root osmotic driving forces play significant roles in plant water uptake (Clarkson et al. 2000) and, in this context, the reduced root osmotic conductance of tos1 (Fig. 8C) could be an important reason for the development of water stress in tos 1 plants.

To summarise, the tos 1 tomato mutant shows lower plant water uptake capacity and a strongly reduced gs and E. Thus, the reduced plant growth of tos 1 plants may be related to competition for water between leaf growth and transpiration, the effects of which are exacerbated with plant development and high evaporative demand. Further explanation of the tos 1 phenotype awaits studies identifying the TOS1 encoding protein, which has been suggested to be an ABA receptor or an early signal transduction component (Borsani et al. 2002).

Acknowledgements - We are grateful to Prof. Jim Syvertsen (Plant Physiolgy, University of Florida) for critically revising the manuscript. We also thank Susan Thurgood and Francisco Bazaga for language corrections on an earlier version of the manuscript. This work was supported in part by the research CICYT-FEDER projects AGL200763610/AGR and AGL2008-01733/AGR (Spain).

\section{References}

Aroca R, Ferrante A, Vernieri P, Chrispeels M (2006) Drought, abcisic and transpiration rate effects on the regulation of PIP acuaporin gene expression and 
abundance in Phaseolus vulgaris plants. Ann Bot 98: 1301-1310

Bray EA (2002) Abcisic acid regulation on gene expression during water-deficit stress in the era of the Arabidopsis genome. Plant Cell Environ 25: 153-161

Borsani O, Cuartero J, Valpuesta V, Botella MA (2002) Tomato tos 1 mutation identifies a gene essential for osmotic tolerance and abcisic acid sensitivity. Plant J 32: 905-914

Chinnusamy V, Shumaker K, Zhu JK (2004) Molecular genetic perspectives on cross-talk and specificity in abiotic stress signalling in plants. J Exp Bot 55: 225-236

Clarkson DT, Carvajal M, Henzler T, Waterhouse RN, Smyth AJ, Cooke DT (2000) Root hydraulic conductance: diurnal aquaporin expression and the effects of nutrient stress. J Exp Bot 51: 61-70

Dodd IC (2005) Root-to-shoot signalling. Assessing the roles of 'up' in the up and down world of long-distance signalling in planta. Plant Soil 274: 251-270

Ebercon A, Blum A, Jordan WR (1977) A rapid colorimetric method for epicuticular wax content of sorghum leaves. Crop Sci 17: 179-180

Ghanem ME, Albacete A, Martínez-Andújar C, Acosta M, Romero-Aranda R, Dodd IC, Lutts S, Pérez-Alfocea F (2008) Hormonal changes during salinity-induced leaf senescent in tomato (Solanum lycopersicum L.) J Exp Bot 59: 3039-3050

Gollan T, Passioura JB, Munns R (1986) Soil water status affects the stomatal conductance of fully turgid wheat and sunflower leaves. Aust J Plant Physiol 13: 459

Grange RI, Hand DW (1987) A review of the effects of atmospheric humidity on the growth of horticultural crops. J Hortic Sci 62: 125-134

Hose E, Staudle E, Hartung W (2000) Abscisic acid and hydraulic conductivity of maize roots: a study using cell- and root- pressure probes. Planta 211: 874-882

Javot H, Maurel C (2002) The role of aquiaporins in root water uptake. Ann Bot 90: 301-313

Karbulková J, Schreiber L, Macek P, Santrucek J (2008) Differences between water permeability of astomatous and stomatous cuticular membranes: effects of air humidity in two species of contrasting drought-resistance strategy. J Exp Bot 59: 3987-3995

Lake JA, Woodward FI (2008) Response of stomatal numbers to $\mathrm{CO}_{2}$ and humidity: control by transpiration rate and abscisic acid. New Phytol 179: 397-404

Mott KA, Denne F, Powell J (1997) Interactions among stomata in response to perturbations in humidity. Plant Cell Environ 20: 1098-1107
Nejad AR, Meeteren U (2007) The role of abscisic acid in disturbed stomatal response characteristics of Tradescantia virginiana during growth at high relative air humidity. J Exp Bot 58: 627-636

Parent B, Hachez C, Redondo E., Simonneau T, Chaumont F, Tardieu F (2009) Drought and abcisic acid effects on aquaporin content translate into changes in hydraulic conductivity and leaf growth rate: a trans-scale approach. Plant Physiol 149: 2000-2012

Pierce M, Raschke K (1980) Correlation between loss of turgor and accumulation of abscisic acid in detached leaves. Planta 148: 174-182

Romero-Aranda R, Soria T, Cuartero J (2001) Tomato plant-water uptake and plant-water relationship under saline growth conditions. Plant Sci 160:

265-272

Romero-Aranda R, Soria T, Cuartero J (2002) Greenhouse mist improves yield of tomato plants grown under saline conditions. J Am Soc Hortic Sci 127: 644-648

Seki M, Umezawa T, Urano K, Shinozaki K (2007) Regulatory metabolic networks in drought stress responses. Curr Opin Plant Biol 10: 1-7

Shinozaki K, Yamaguchi-Shinozaki K (2007) Gene networks involved in drought stress response and tolerance. J Exp Bot 58: 221-227

Steudle E, Peterson CA (1998) How does water get through roots? J Exp Bot 49: 775-788

Tardieu F, Davies WJ (1992) Stomatal response to abscisic acid is a function of current plant water status. Plant Physiol 98: 540-549

Urano K, Maruyama K, Ogata Y, Morishita Y, Takeda M, Sakurai N, Suzuki H, Saito K, Shibata D, Kobayashi M, Yamaguchi-Shinozaki K, Shinozaki K (2009) Characterization of the ABA-regulated global responses to dehydration in Arabidopsis by metabolomics. Plant J 57: 1065-1078

Vysotskaya LB, Koroboba AV, Kudoyarova GR (2008) Abcisic acid accumulation in the roots of nutrient-limited plants: Its impact on the differential growth of roots and shoots. J Plant Physiol 165: 1274-1279

Wilkinson S, Davies WJ (2002) ABA-based chemical signalling: the co-ordination of responses to stress in plants. Plant Cell Environ 25: 195-210

Yao C, Moreshet S, Aloni B (2001) Water relations and hydraulic control of stomatal behaviour in bell pepper plant in partial soil drying. Plant Cell Environ 12: $73-81$ 